\title{
Left atrial appendage elimination techniques: stapled excision versus internal suture obliteration
}

\author{
Yoonjin Kang^, Ho Young Hwang, Seohee Joo, Ji Hyeon Park, Ji Seong Kim, Suk Ho Sohn^, \\ Jae Woong Choi
}

Department of Thoracic and Cardiovascular Surgery, Seoul National University Hospital, Seoul, Republic of Korea

Contributions: (I) Conception and design: HY Hwang; (II) Administrative support: HY Hwang; (III) Provision of study materials or patients: HY Hwang, Y Kang, JS Kim, SH Sohn, JW Choi; (IV) Collection and assembly of data: Y Kang, HY Hwang, S Joo, JH Park, JW Choi (V) Data analysis and interpretation: Y Kang, HY Hwang; (VI) Manuscript writing: All authors; (VII) Final approval of the manuscript: All authors.

Correspondence to: Ho Young Hwang, MD, PhD. Department of Thoracic and Cardiovascular Surgery, Seoul National University Hospital, Seoul National College of Medicine, 101 Daehak-ro, Chongno-gu, Seoul 03080, Republic of Korea. Email: scalpel@hanmail.net.

Background: This study was conducted to compare the efficacy and safety of left atrium appendage (LAA) elimination using stapled excision with those using internal suture obliteration.

Methods: In all, 158 patients with atrial fibrillation who underwent surgical elimination of the LAA during cardiac surgery and later underwent postoperative computed tomography (CT) were enrolled. Of these, 102 patients underwent stapled excision (SE group), and 56 underwent internal suture obliteration (IO group). The efficacy endpoint was LAA excision failure, including a remnant LAA $(>1 \mathrm{~cm})$ or communication between the left atrium (LA) and LAA. The safety endpoint was bleeding from the elimination site. The CT was performed at a median of 12.8 months (interquartile range, 0.3-39.9) after surgery.

Results: The efficacy endpoint was observed in 5 (4.9\%) and 9 patients (16.1\%) in the SE and IO groups, respectively $(\mathrm{P}=0.036)$. In the $\mathrm{SE}$ group, a remnant LAA was found in 5 patients and extravasation of the dye was not observed. In the IO group, a remnant LAA, a communication between the LA and LAA, and both findings were observed in 1, 6, and 2 patients, respectively. The safety endpoint occurred in $12(11.8 \%)$ and 0 patients $(0 \%)$ in the SE and IO groups, respectively $(\mathrm{P}=0.009)$.

Conclusions: Stapled excision of the LAA was associated with a lower rate of LAA elimination failure compared to internal suture obliteration. However, care should be taken to prevent staple-related bleeding.

Keywords: Cardiac; arrhythmia therapy; surgical equipment

Submitted Jul 09, 2021. Accepted for publication Sep 27, 2021.

doi: $10.21037 /$ jtd-21-1138

View this article at: https://dx.doi.org/10.21037/jtd-21-1138

\section{Introduction}

Atrial fibrillation (AF) is the most prevalent arrhythmia and an independent risk factor for stroke and systemic thromboembolism (1). In AF patients, the overall risk of stroke per year is reported to be $5 \%(2,3)$. The risk of stroke is age-dependent, being about 16 -fold higher in $\mathrm{AF}$ patients in their 50 s than those in their 80 s (3). The left atrium appendage (LAA) is an important source of thrombi in AF patients (4). Therefore, elimination of the LAA may be a key procedure in AF patients who undergo cardiac surgery to reduce further thromboembolic events (5-7).

Surgical LAA elimination techniques include surgical excision, stapled excision, internal suture obliteration and epicardial clip occlusion $(8,9)$. Previous studies have demonstrated controversial results regarding the safety

^ ORCID: Yoonjin Kang, 0000-0002-2528-173X; Suk Ho Sohn, 0000-0001-7391-3415. 
Table 1 Preoperative data of the study patients

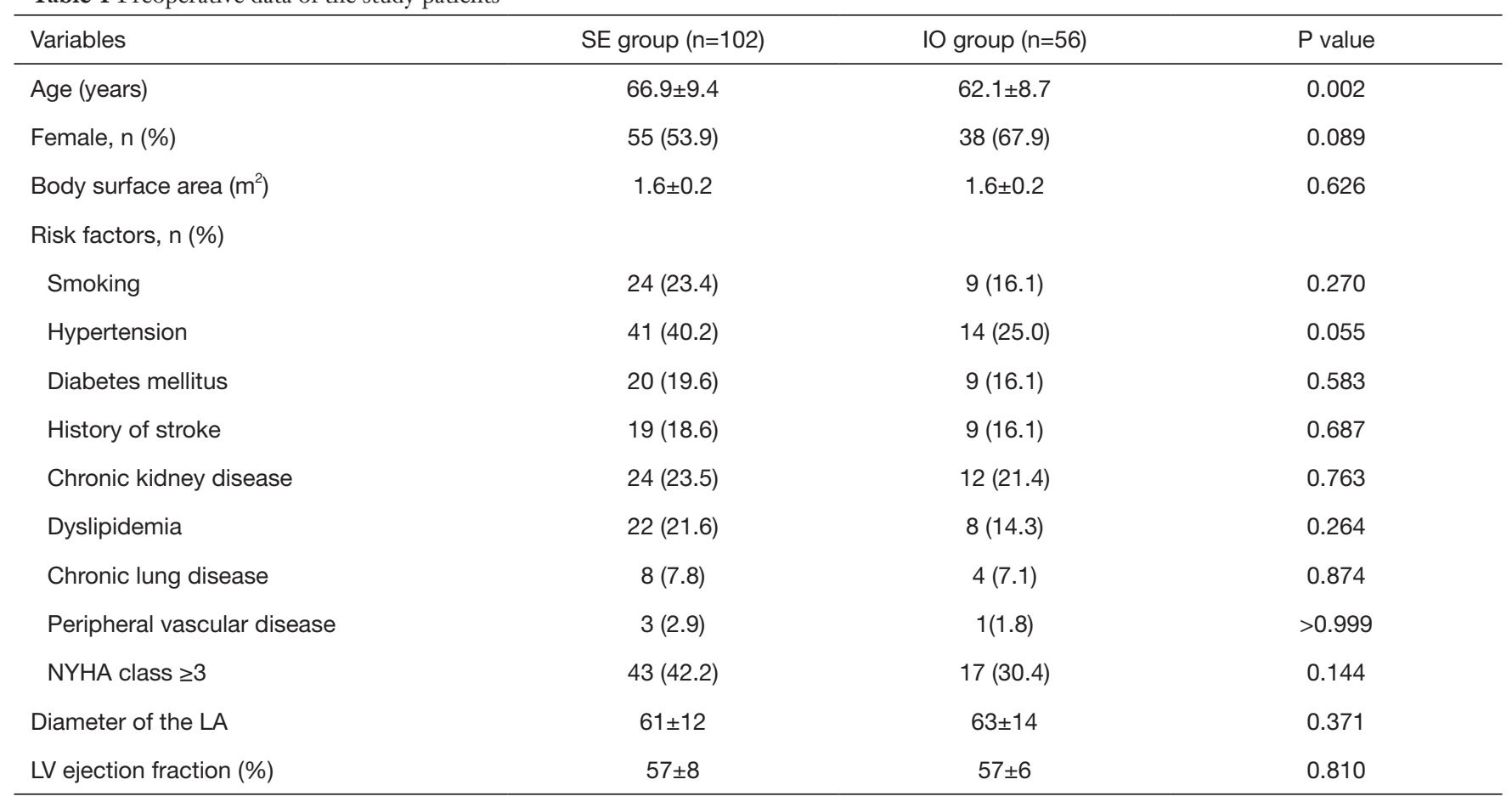

Values are $\mathrm{n}(\%)$ or mean \pm SD. NYHA, New York Heart Association; LA, left atrium; LV, left ventricle.

and efficacy of various LAA elimination techniques (9-12). Therefore, this study compared the safety and efficacy of stapled excision and internal suture obliteration of the LAA.

We present the following article in accordance with the STROBE reporting checklist (available at https://dx.doi. org/10.21037/jtd-21-1138).

\section{Methods}

\section{Patient characteristics}

The study protocol was reviewed by the Institutional Review Board of our institution and approved as a minimalrisk retrospective study and individual consent was waived (approval no. H-2101-025-1185). The study was conducted in accordance with the Declaration of Helsinki (as revised in 2013). Between January 2013 and February 2020, a total of 234 AF patients underwent LAA elimination during concomitant cardiac surgery. Of these patients, 10 patients who underwent previous cardiac surgery were excluded. A total of 158 patients (male:female, 65:93) who later underwent postoperative computed tomography (CT) were enrolled in the this study. Of these, 102 patients underwent LAA elimination using stapled excision (SE group) and
56 patients with internal suture obliteration (IO group). A total of 49 patients of the stapled excision group in the previous study (12) were included in this study. All enrolled patients were included in the analyses. The mean age at the surgery was $65.2 \pm 9.4$ years. The median duration of $\mathrm{AF}$ was 3.4 years (interquartile range, $0.3-9.0$ years). Hypertension $(34.8 \%)$ and chronic kidney disease $(22.8 \%)$ were the most common comorbidities. The SE group patients were older than the IO group $(\mathrm{P}=0.002)$ (Table 1).

\section{Surgical procedures, operative data and postoperative anticoagulation}

All procedures were performed through a median sternotomy, with aorto-bicaval cannulation under moderate hypothermia and cold cardioplegic arrest. Neither the morphology of the LAA nor positional relationship of LA and LAA was criteria to avoid stapled excision. The choice of LAA elimination technique was according to each surgeon's preference; stapled excision was performed by two surgeons having hundreds of experiences in valvular heart surgery, and internal suture obliteration was performed by one senior surgeon having thousands of experience in valvular heart surgery. 
Table 2 Operative data of the study patients

\begin{tabular}{lccc}
\hline Variables & SE group $(\mathrm{n}=102)$ & IO group $(\mathrm{n}=56)$ & $\mathrm{P}$ value \\
\hline CPB time (minutes) & $171 \pm 56$ & $240 \pm 65$ & $<.001$ \\
ACC time (minutes) & $117 \pm 38$ & & $<.001$ \\
Concomitant procedure, $\mathrm{n}(\%)$ & & $21(37.5)$ & 0.114 \\
Aortic valve surgery & $26(25.5)$ & $49(87.5)$ & 0.003 \\
Mitral valve surgery & $67(65.7)$ & $29(51.8)$ & 0.356 \\
Tricuspid valve surgery & $45(44.1)$ & $47(83.9)$ & 0.339 \\
Arrhythmia surgery & $91(89.2)$ & $0(0.0)$ & 0.052 \\
Coronary artery bypass grafting & $7(6.9)$ & & \\
\hline
\end{tabular}

Values are $\mathrm{n}(\%)$ or mean \pm SD. CPB, cardiopulmonary bypass; ACC, aortic cross clamp.

All of the patients in the SE group underwent LAA excision with an Endo GIA Vascular Stapler (Medtronic, Minneapolis, MN, USA) using a single $60 \mathrm{~mm}$ cartridge $(\mathrm{n}=84)$ or both $60 \mathrm{~mm}$ and additional $45 \mathrm{~mm}$ cartridges $(\mathrm{n}=18)$. Purple-colored cartridges were used in the most of patients $(n=48,30.4 \%)$. In the middle of study periods, Tan-colored cartridge was used in $\mathrm{xxx}$ following thoracic surgeons' advice $(\mathrm{n}=53,33.5 \%)$. Since LAA excision technique using staplers were adopted, details about bleeding and reinforcement sutures have been recorded in our institutional database. In the IO group, internal suture obliteration was performed with a double-layer continuous suture or multiple mattress interrupted sutures reinforced with pledgets. The LAA was not excised in any patients. LAA excision or internal suture obliteration was performed after the completion of all other procedures, before closing the left atriotomy.

Mean cardiopulmonary bypass $(\mathrm{CPB})$ and aortic cross clamp (ACC) times were $196 \pm 67$ and $137 \pm 52$ minutes, respectively. All patients underwent either mitral valve surgery $(\mathrm{n}=116)$ or maze procedure $(\mathrm{n}=138)$. Other cardiac procedures included tricuspid valve surgery $(\mathrm{n}=74)$, aortic valve operation $(n=48)$ and coronary artery bypass grafting $(\mathrm{n}=7)$. The CPB and ACC times were significantly longer and concomitant mitral valve related procedures were more frequently performed in the IO than SE group (Table 2).

Low-molecular-weight heparin was initiated postoperatively in all patients, usually on postoperative day 1 and overlapped with warfarin anticoagulation. The target international normalized ratio ranged from 2.0 to 3.0 based on the types of concomitant procedures. Aspirin $(100 \mathrm{mg} /$ day $)$ was added in 7 patients who underwent concomitant coronary artery bypass grafting.

\section{Evaluation of early clinical outcomes}

Operative mortality was defined as death within 30 days of the operation or during the same hospitalization. Postoperative low cardiac output syndrome was defined as the need for mechanical supports or inotropic support to maintain systolic blood pressure $>90 \mathrm{mmHg}$ even after correction of reversible factors. Respiratory complications included postoperative pneumonia or $>48$ hours of prolonged ventilator support. Acute kidney injury was defined as an increase of $>50 \%$ in serum creatinine from the base level according to the Risk, Injury, Failure, Loss of kidney function, and End-stage kidney disease criteria (13). Postoperative stroke, either hemorrhagic or embolic, was defined as any new neurological deficit presenting in hospital with accompanying neuroimaging confirmation (CT or brain magnetic resonance imaging), and diagnostic confirmation by a neurologist. Other thromboembolic events were defined as systemic thromboembolic events other than a stroke or transient ischemic attack.

\section{Evaluation of the left atrial appendage using computed tomography}

For the evaluation of the LAA, electrocardiogram-gated cardiac CT scans were performed with dual-source CT (Somatom Force; Siemens Medical Solutions, Forchheim, Germany). Image acquisition was gated to $40 \%$ of the $\mathrm{RR}$ interval. The contrast injection protocol consisted of $70 \mathrm{~mL}$ of iopromide (Iomeron 400; Bracco Diagnostics, Milan, Italy) followed by $50 \mathrm{~mL}$ of an 8:2 mixture of normal saline and contrast medium at a flow rate of $4 \mathrm{~mL} / \mathrm{sec}$. 
Table 3 Early clinical outcomes

\begin{tabular}{lccc}
\hline Variables & SE group $(\mathrm{n}=102)$ & IO group $(\mathrm{n}=56)$ & 0.539 \\
\hline Operative mortality, $\mathrm{n}(\%)$ & $2(2.0)$ & & \\
Complications, $\mathrm{n}(\%)$ & & & \\
Acute kidney injury & $32(31.4)$ & $11(19.6)$ & 0.113 \\
LCOS & $11(10.8)$ & $11(19.6)$ & 0.124 \\
Respiratory complication & $12(11.8)$ & $1(10.7)$ & 0.842 \\
Bleeding reoperation & $8(7.8)$ & $0(0.0)$ & 0.160 \\
Hemorrhagic stroke & $0(0.0)$ & $2(3.6)$ & $>0.999$ \\
Embolic stroke & $2(2.0)$ & $0(0.0)$ & 0.615 \\
Mediastinitis & $0(0.0)$ & & $>0.999$ \\
\hline
\end{tabular}

LCOS, low cardiac output syndrome.

Gated cardiac CT scans were performed at first pass and 2 minutes after contrast administration. One radiologist, who was specialized for cardiovascular imaging, reviewed all the CT scans. The median interval between the operation and CT scan was 12.8 months (interquartile range $0.3-39.9$ months). All enrolled patients underwent CT scanning.

Patients with decreased renal function underwent contrast enhanced CT scans with pre- and post-scan managements to prevent aggravation of renal dysfunction with a consultation to a nephrologist.

\section{Efficacy and safety endpoints of left atrial appendage elimination}

The primary efficacy endpoint was LAA elimination failure. In the SE group, this was defined as the presence of a remnant LAA (an LAA pouch with a maximum length $>1 \mathrm{~cm}$ on three dimensional CT scan) or extravasation of radiocontrast dye outside the LA. In the IO group, it was defined as the presence of a remnant LAA (an LAA pouch with a maximum length $>1 \mathrm{~cm}$ on three dimensional CT scan) or residual communication between the LA and obliterated LAA. In addition, the presence of a LA thrombus was evaluated as the secondary efficacy endpoint.

The safety endpoint was defined as bleeding from the elimination site requiring reinforcement sutures during surgery or reoperation.

\section{Statistical analysis}

Statistical analyses were performed using the SPSS software package (version 23.0; IBM Corp., Armonk, NY, USA). Data are expressed as the mean \pm standard deviation, median with interquartile range, or a proportion. Comparisons between the two groups were performed using the chisquare test or Fisher's exact test and Student's $t$-test for categorical and continuous variables, respectively. The data were assumed to follow normal distribution as the sample size was greater than 30 based on central limit theorem. Fisher's test was adopted when $25 \%$ of expected frequencies is less than or equal to 5 in $2 \times 2$ contingency table. A P value $<0.05$ was considered as statistically significant.

\section{Results}

\section{Early clinical outcomes}

Early mortality occurred in 2 and 0 patients in the SE and IO groups, respectively. One patient died of aggravation of ischemic colitis which was present preoperatively on the $23 \mathrm{rd}$ postoperative day. The other patient expired due to septic shock on the 13th postoperative day. Postoperative complications included acute kidney injury $(\mathrm{n}=43,27.2 \%)$, low cardiac output syndrome $(\mathrm{n}=22,13.9 \%)$, respiratory complications ( $\mathrm{n}=18,11.4 \%)$, postoperative bleeding requiring reoperation $(n=9,5.7 \%)$ and stroke $(n=4,2.5 \%)$. There were no statistically significant differences in operative mortality or postoperative complications between the two groups (Table 3).

\section{Evaluation of efficacy endpoint}

The CT scans of the patients with successful LAA 

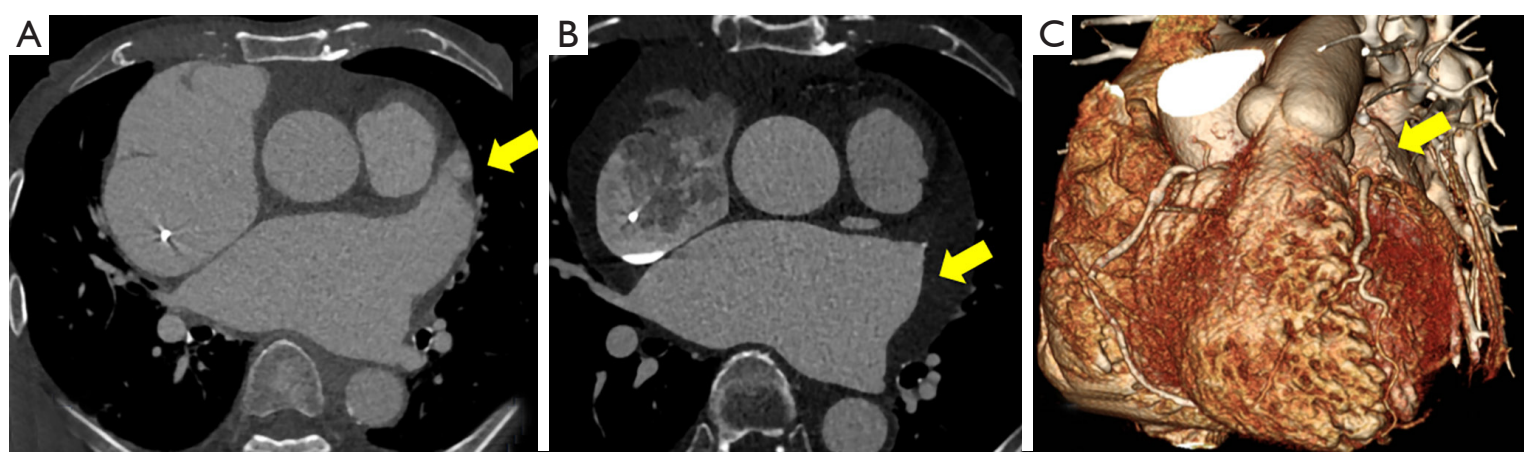

Figure 1 A case of successful left atrial appendage (LAA) elimination in the stapled excision group. (A) Preoperative electrocardiogramgated computed tomography (CT) scan showing LAA. (B) A two-dimensional and (C) three-dimensional postoperative CT scan showing a completely excised LAA. The arrow indicates well excised LAA.
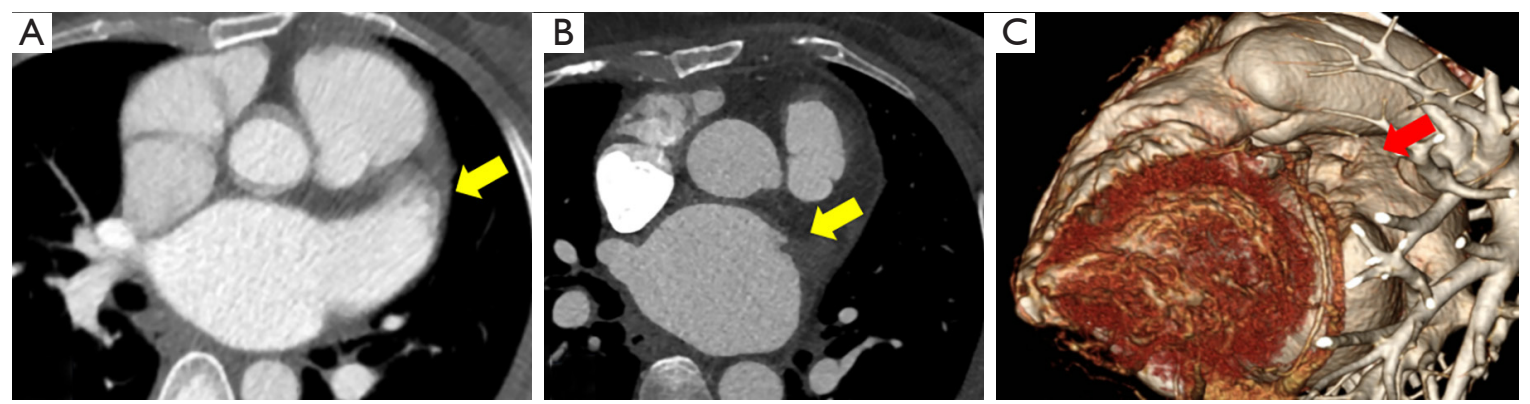

Figure 2 A case of successful left atrial appendage (LAA) elimination in the internal suture obliteration group. (A) preoperative electrocardiogram-gated computed tomography (CT) scan showing LAA. (B) A two-dimensional and (C) three-dimensional postoperative CT scan showing a completely obliterated LAA. The arrow indicates well obliterated LAA.
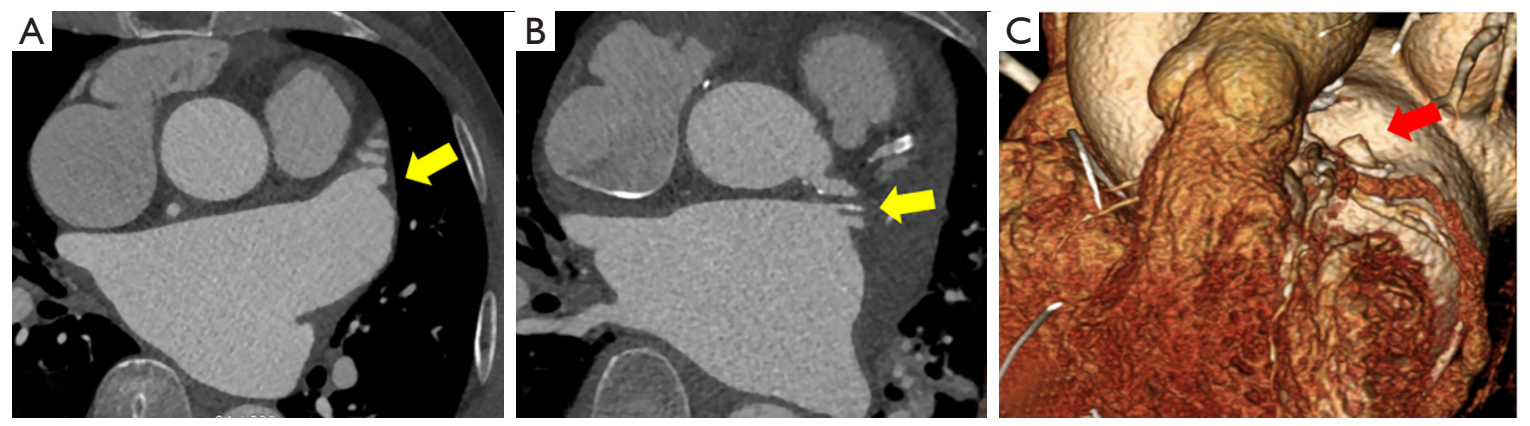

Figure 3 A case of left atrial appendage (LAA) elimination failure in the stapled excision group. (A) Preoperative electrocardiogram-gated computed tomography (CT) scan showing LAA. (B) A two-dimensional and (C) three-dimensional postoperative CT scan showing LAA remnant after stapled excision. The arrow indicates remnant LAA.

elimination in the $\mathrm{SE}$ and $\mathrm{IO}$ groups are presented in Figures 1,2, respectively. A remnant LAA was seen in 5 (4.9\%) patients in the SE group (Figure 3). Extravasation of radiocontrast dye was not found in any patients. In the IO group, a LAA remnant, residual communication between the LA and LAA, and both findings were seen in 1, 6, and 2 patients, respectively (Figure 4). The occurrence rate of the efficacy endpoint was significantly lower in the SE than in 

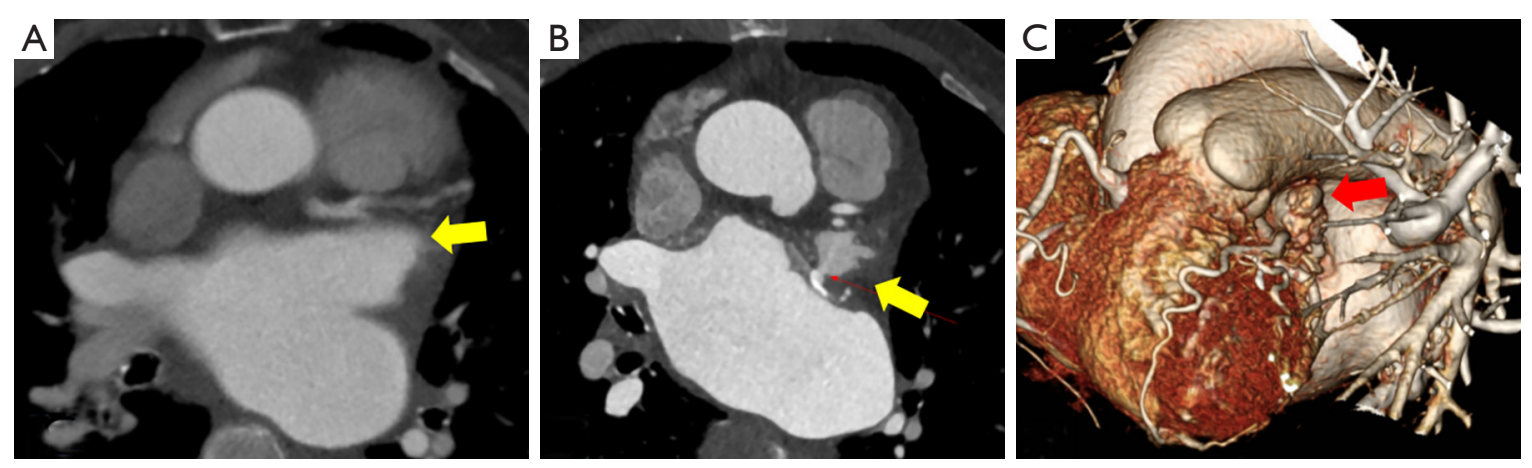

Figure 4 A case of left atrial appendage (LAA) elimination failure in the internal suture obliteration (IO) group. (A) Preoperative electrocardiogram-gated computed tomography (CT) scan showing LAA. (B) A two-dimensional and (C) three-dimensional postoperative CT scans showing incompletely obliterated LAA. Residual communication between the left atrium and LAA are shown. The arrow indicates incompletely obliterated LAA.

Table 4 Occurrence rates of efficacy and safety endpoints

\begin{tabular}{|c|c|c|c|}
\hline Variables & SE group $(n=102)$ & IO group $(n=56)$ & $P$ value \\
\hline LAA remnant & $5(4.9)$ & $1(1.8)$ & \\
\hline Residual communication (or extravasation of dye) & $0(0)$ & $6(10.7)$ & \\
\hline Both findings & $0(0)$ & $2(3.6)$ & \\
\hline Safety endpoint (= elimination site bleeding), n (\%) & $12(11.8)$ & $0(0)$ & 0.009 \\
\hline Control during index operation & $12(11.8)$ & $0(0)$ & \\
\hline Staple line bleeding & $8(7.8)$ & - & \\
\hline Bleeding from surrounding tissues & $4(3.9)$ & - & \\
\hline
\end{tabular}

LAA, left atrium appendage; LA, left atrium.

the $\mathrm{IO}$ groups ( $4.9 \%$ vs. $16.1 \%, \mathrm{P}=0.036)$ (Table 4).

In addition, LA thrombus was found in one patient in the LAAE group and target international normalized ratio was increased in this patient.

\section{Occurrence of safety endpoint}

In the SE group, bleeding from the LAA elimination site was controlled intraoperatively in 12 patients $(11.8 \%)$. There were $8(7.8 \%)$ cases of bleeding from the staple line and $4(3.9 \%)$ cases of bleeding from surrounding tissues requiring reinforcement sutures (Table 4). Occurrence of bleeding events were not associated with the types of cartridge $(\mathrm{P}=0.734)$. No patient in the IO group had bleeding from the LAA elimination site. No patient in either group required reoperation due to LAA elimination site bleeding. The occurrence rate of the safety endpoint was significantly higher in the SE than in the IO group (11.8\% vs. $0 \%, \mathrm{P}=0.009)$.

\section{Discussion}

The present study demonstrates two main findings. First, stapled excision of the LAA resulted in better results in terms of complete elimination of the LAA than internal suture obliteration. Second, stapled excision of the LAA was associated with a fairly high rate of excision site bleeding due to staple line disruption or tears in the surrounding 
epicardial tissues.

The elimination of the LAA has been considered as a key procedure in AF patients who undergo cardiac surgery to reduce thromboembolic events $(5,6)$. Recently, a randomized controlled clinical trial called 'LAAOS III trial' (7) confirmed this advantage once again; in this trial, 2,379 AF patients with CHA2DS2-VASc score equal or over 2 were randomly assigned to undergo or not undergo LAA elimination during cardiac surgery. The study results showed that the risk of ischemic stroke or systemic embolism was lower in LAA elimination group than the other group. Various surgical techniques have been proposed for the elimination of the LAA during cardiac surgery to prevent thromboembolic events associated with AF. These include an external approach such as suture ligation, stapled or surgical excision, and an internal approach such as suture obliteration $(8,9,14)$. Previous studies have demonstrated the advantages and disadvantages of each technique. When considering effectiveness, complete exclusion of the LAA from bloodstream may be important to prevent a late stroke or systemic embolization $(6,9)$.

Although internal suture obliteration of the LAA may be a simple and inexpensive choice for LAA exclusion, it is associated with incomplete obliteration in 10-30\% of patients (11), predisposing them to thromboembolic events (15). Another study (10) showed that stapled excision resulted in $90 \%$ complete LAA elimination. However, comparative analyses between those two elimination techniques are limited. Despite surgeons' common sense that the LAA excision using staple might be a good option for LAA elimination, previous studies have demonstrated unsatisfactory results of the LAA excision. A previous randomized controlled study compared the effectiveness of internal ligation, stapled excision and surgical excision in 28 patients (9). The overall failure of LAA elimination was around $57 \%$ without any significant differences among the three groups. However, the study was limited due to the sample size being too small to detect a possible difference.

Thus, as a continuum of our previous study (12), this study compared the efficacy of stapled excision and internal suture obliteration in 168 patients, which was relatively large patient population compared to previous studies $(9,11)$. A total of 50 patients of the stapled excision group in the previous study were included in this study. We found that stapled excision of the LAA during cardiac surgery resulted in complete elimination in more than $95 \%$ of patients. This is consistent with findings of a previous study showing a
$90 \%$ success rate after stapled excision (10). However, in contrast to the favorable efficacy results, this study also showed an association with a high rate of stapled excisionrelated bleeding directly from the staple line or from surrounding tissues, as reported previously (12), whereas no safety events occurred after internal suture obliteration. Because the LAA is not a linear structure, has variable morphology, and has a thin wall at the base, there may a risk of vascular staple line disruption. In addition, manipulation of the bulky vascular stapler in a narrow pericardial space might result in soft tissue tears around the LAA.

The epicardial clip is recently introduced device for LAA elimination, and the safety and efficacy of this technique has been reported $(16,17)$. However, low flexibility and relatively bulky size of the device makes it difficult to use in a specific angulation or configuration (18). In addition, it is not popularized yet in many countries including ours. In our country, the use of the epicardial clip is very limited and it is insurance-covered only in cases of minimally invasive cardiac surgery.

Previous studies used transesophageal echocardiography (TEE) to evaluate the results of surgical LAA elimination. Although TEE is an effective tool for visualization of the LA and LAA, it is an invasive and costly procedure. Recently, non-invasive cardiac CT scans have been used to evaluate LAA elimination sites or intracardiac thrombi, and cardiac CT is a reasonable alternative to TEE $(19,20)$. Cardiac CT became a routine follow-up protocol after surgical elimination of the LAA at our institution during the later study period. Therefore, patients in the early study period underwent CT scans at a longer time period after surgery than those in the later study period.

The present study has several limitations. First, this was a retrospective study performed at a single institution. Second, not all patients who underwent surgical LAA elimination underwent a subsequent CT scan. Third, the baseline characteristics between the two groups were not identical. Although, selection of the elimination technique was based on the surgeon's preference rather than patient characteristics, these differences might have affected our study results. The results were compared using univariate analyses because the effect of baseline characteristics on the study results, particularly the efficacy endpoint, may be minimal. Moreover, our analysis did not include other elimination techniques including epicardial clip.

In addition, follow-up clinical outcomes related to $\mathrm{AF}$ such as thromboembolism and bleeding were not evaluated because the study only included patients who underwent 
CT scans after surgery. The number of events (data not shown) was too small to draw any relevant longitudinal outcome data. Finally, the different intervals between the index operation to CT scan, which is inevitable as a retrospective study, are one of the limitations of the present study.

\section{Conclusions}

Stapled excision of the LAA was associated with a lower rate of LAA elimination failure compared to internal suture obliteration. However, cautious handling of the vascular stapler is recommended to prevent LAA excisionrelated bleeding from staple line disruption or injury to surrounding soft tissues.

\section{Acknowledgments}

Funding: None.

\section{Footnote}

Reporting Checklist: The authors have completed the STROBE reporting checklist. Available at https://dx.doi. org/10.21037/jtd-21-1138

Data Sharing Statement: Available at https://dx.doi. org/10.21037/jtd-21-1138

Conflicts of Interest: All authors have completed the ICMJE uniform disclosure form (available at https://dx.doi. org/10.21037/jtd-21-1138). The authors have no conflicts of interest to declare.

Ethical Statement: The authors are accountable for all aspects of the work in ensuring that questions related to the accuracy or integrity of any part of the work are appropriately investigated and resolved. The study was conducted in accordance with the Declaration of Helsinki (as revised in 2013). The study protocol was reviewed by the Institutional Review Board of our institution and approved as a minimal-risk retrospective study and individual consent was waived (approval No.: H-2101-025-1185).

Open Access Statement: This is an Open Access article distributed in accordance with the Creative Commons Attribution-NonCommercial-NoDerivs 4.0 International License (CC BY-NC-ND 4.0), which permits the non- commercial replication and distribution of the article with the strict proviso that no changes or edits are made and the original work is properly cited (including links to both the formal publication through the relevant DOI and the license). See: https://creativecommons.org/licenses/by-nc-nd/4.0/.

\section{References}

1. Caliskan E, Cox JL, Holmes DR Jr, et al. Interventional and surgical occlusion of the left atrial appendage. Nat Rev Cardiol 2017;14:727-43.

2. Kanderian AS, Gillinov AM, Pettersson GB, et al. Success of surgical left atrial appendage closure: assessment by transesophageal echocardiography. J Am Coll Cardiol 2008;52:924-9.

3. Wolf PA, Abbott RD, Kannel WB. Atrial fibrillation as an independent risk factor for stroke: the Framingham Study. Stroke 1991;22:983-8.

4. Blackshear JL, Odell JA. Appendage obliteration to reduce stroke in cardiac surgical patients with atrial fibrillation. Ann Thorac Surg 1996;61:755-9.

5. García-Fernández MA, Pérez-David E, Quiles J, et al. Role of left atrial appendage obliteration in stroke reduction in patients with mitral valve prosthesis: a transesophageal echocardiographic study. J Am Coll Cardiol 2003;42:1253-8.

6. Aryana A, Singh SK, Singh SM, et al. Association between incomplete surgical ligation of left atrial appendage and stroke and systemic embolization. Heart Rhythm 2015;12:1431-7.

7. Whitlock RP, Belley-Cote EP, Paparella D, et al. Left Atrial Appendage Occlusion during Cardiac Surgery to Prevent Stroke. N Engl J Med 2021;384:2081-91.

8. Turagam MK, Velagapudi P, Kar S, et al. Cardiovascular Therapies Targeting Left Atrial Appendage. J Am Coll Cardiol 2018;72:448-63.

9. Lee R, Vassallo P, Kruse J, et al. A randomized, prospective pilot comparison of 3 atrial appendage elimination techniques: Internal ligation, stapled excision, and surgical excision. J Thorac Cardiovasc Surg 2016;152:1075-80.

10. Healey JS, Crystal E, Lamy A, et al. Left Atrial Appendage Occlusion Study (LAAOS): results of a randomized controlled pilot study of left atrial appendage occlusion during coronary bypass surgery in patients at risk for stroke. Am Heart J 2005; 150:288-93.

11. Katz ES, Tsiamtsiouris T, Applebaum RM, et al. Surgical left atrial appendage ligation is frequently incomplete: a transesophageal echocardiograhic study. J Am Coll Cardiol 
2000;36:468-71.

12. Park JH, Sohn SH, Choi JW, et al. Safety and Efficacy of Left Atrial Appendage Excision Using a Vascular Stapler. Korean J Thorac Cardiovasc Surg 2020;53:127-31.

13. D'Onofrio A, Cruz D, Bolgan I, et al. RIFLE criteria for cardiac surgery-associated acute kidney injury: risk factors and outcomes. Congest Heart Fail 2010;16 Suppl 1:S32-6.

14. Greenberg JW, Lee R, Hui DS. Patient selection and methods of surgical left atrial appendage exclusion. J Thromb Thrombolysis 2019;48:209-14.

15. Rosenzweig BP, Katz E, Kort S, et al. Thromboembolus from a ligated left atrial appendage. J Am Soc Echocardiogr 2001;14:396-8.

16. Salzberg SP, Plass A, Emmert MY, et al. Left atrial appendage clip occlusion: early clinical results. J Thorac Cardiovasc Surg 2010;139:1269-74.

Cite this article as: Kang Y, Hwang HY, Joo S, Park JH, Kim JS, Sohn SH, Choi JW. Left atrial appendage elimination techniques: stapled excision versus internal suture obliteration. J Thorac Dis 2021;13(11):6252-6260. doi: 10.21037/jtd-21-1138
17. Kurfirst V, Mokrácek A, Canádyová J, et al. Epicardial clip occlusion of the left atrial appendage during cardiac surgery provides optimal surgical results and long-term stability. Interact Cardiovasc Thorac Surg 2017;25:37-40.

18. Suematsu Y, Shimizu T. Clip-and-loop technique for left atrial appendage occlusion. Asian Cardiovasc Thorac Ann 2020;28:618-20.

19. Aimo A, Kollia E, Ntritsos G, et al. Echocardiography versus computed tomography and cardiac magnetic resonance for the detection of left heart thrombosis: a systematic review and meta-analysis. Clin Res Cardiol 2021;110:1697-703.

20. Hur J, Kim YJ, Lee HJ, et al. Left atrial appendage thrombi in stroke patients: detection with two-phase cardiac CT angiography versus transesophageal echocardiography. Radiology 2009;251:683-90. 